\title{
Lower Eyelid Blepharoplasty
}

Roger L. Crumley, Behrooz A. Torkian, and Amir M. Karam

\section{Anatomical Considerations}

In no other area of facial aesthetic surgery is such a fragile balance struck between form and function as that in eyelid modification. Owing to the delicate nature of eyelid structural composition and the vital role the eyelids serve in protecting the visual system, iatrogenic alterations in eyelid anatomy must be made with care, precision, and thoughtful consideration of existing soft tissue structures. A brief anatomical review is necessary to highlight some of these salient points.

With the eyes in primary position, the lower lid should be well apposed to the globe, with its lid margin roughly tangent to the inferior limbus and the orientation of its respective palpebral fissure slanted slightly obliquely upward from medial to lateral (occidental norm). An inferior palpebral sulcus (lower eyelid crease) is usually identified $\sim 5$ to $6 \mathrm{~mm}$ from the ciliary margin and roughly delineates the inferior edge of the tarsal plate and the transition zone from pretarsal to preseptal orbicularis oculi ${ }^{1}$ (Fig. 23.1).

\section{Lamellae}

The eyelids have been considered as being composed of two lamellae: (1) an outer lamella, composed of skin and

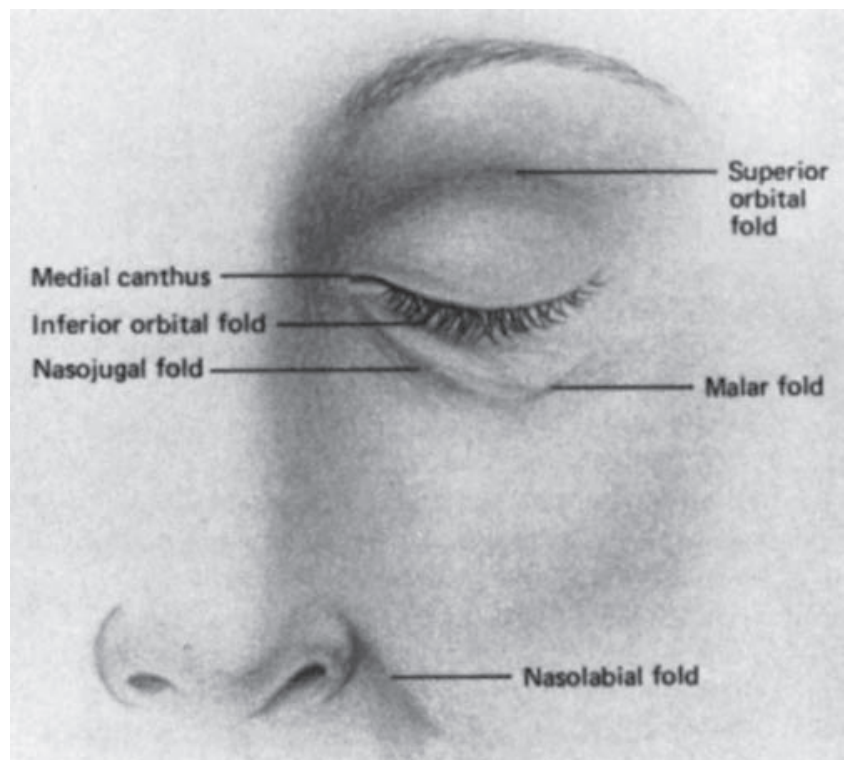

Fig. 23.1 Topographic anatomy of the eyelid, demonstrating natural folds. (From Kohn R. Textbook of Ophthalmic Plastic and Reconstructive Surgery. Philadelphia: Lea \& Febiger; 1988. Reprinted by permission.) the orbicularis oculi muscle and (2) an inner lamella, which includes tarsus and conjunctiva. The skin of the lower eyelid, which measures less than $1 \mathrm{~mm}$ in thickness, ${ }^{2}$ retains a smooth delicate texture until it extends beyond the lateral orbital rim, where it gradually becomes thicker and coarser. The eyelid skin, which is essentially devoid of a subcutaneous fat layer, is interconnected to the underlying musculus orbicularis oculi by fine connective tissue attachments in the skin's pretarsal and preseptal zones.

\section{Musculature}

The orbicularis oculi muscle can be divided into a darker and thicker orbital portion (voluntary) and a thinner and lighter palpebral portion (voluntary and involuntary). The palpebral portion can be further subdivided into preseptal and pretarsal components (Fig. 23.2). The larger superficial heads of the pretarsal orbicularis unite to form the medial canthal tendon, which inserts onto the anterior lacrimal crest, whereas the deep heads unite to insert at the posterior lacrimal crest. Laterally, the fibers condense and become firmly attached at the orbital tubercle of Whitnall, becoming the lateral canthal tendon. ${ }^{3}$ Although the preseptal orbicularis has fixed attachments with the medial and lateral canthal tendons, the orbital portion does not and instead inserts subcutaneously in the lateral orbital region (contributing to crow's feet), overlies some of the elevator muscles of the upper lip and ala, and possesses attachments to the infraorbital bony margin.

Immediately beneath the submuscular fascia, extending along the posterior surface of the preseptal orbicularis, lies the orbital septum. Delineating the boundary between anterior eyelid (outer lamella) and intraorbital contents, it originates at the arcus marginalis along the orbital rim (continuous with orbital periosteum), and after fusing with the capsulopalpebral fascia posteriorly $\sim 5 \mathrm{~mm}$ below the lower tarsal edge, it forms a single fascial layer that inserts near the tarsal base.

The capsulopalpebral head of the inferior rectus is a dense, fibrous, connective tissue expansion, which by virtue of its ultimate attachments to the tarsal plate allows for lower lid retraction during downward gaze. During its forward extension, it encompasses the musculus obliquus inferior, and after reuniting anteriorly, it contributes to the formation of Lockwood's suspensory ligament (inferior transverse ligament, at which point it is termed the capsulopalpebral fascia, $\mathrm{CPF}) .{ }^{4}$ Although the majority of its fibers terminate near the 


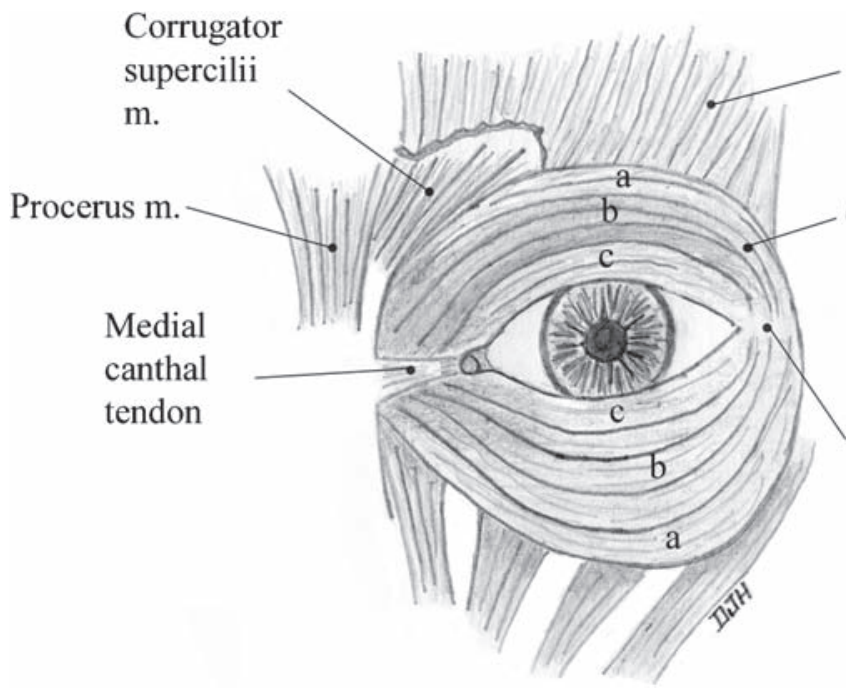

inferior tarsal border, some extend through orbital fat contributing to compartmentalization, some penetrate the preseptal orbicularis to insert subcutaneously about the lower eyelid crease, and others extend from the inferior fornix superiorly to contribute to Tenon's capsule (Fig. 23.3).

\section{Orbital Fat}

Contained behind the orbital septum and within the orbital cavity, the orbital fat has been classically segmented into discrete pockets (lateral, central, and medial), although interconnections truly exist. ${ }^{5}$ The lateral fat pad is smaller and more superiorly situated, and the larger nasal pad is divided by the inferior oblique muscle into a larger central fat compartment and an intermediate medial compartment. (During surgery, care must be taken to avoid injury to the inferior oblique.) The medial pad has characteristic
Frontalis m.

Orbicularis oculi m.
a. preorbital
b. preseptal
c. pretarsal

Lateral

palpebral

raphe

Fig. 23.2 Major divisions of the $\mathrm{m}$. orbicularis oculi into pretarsal, preseptal, and orbital components. differences from its other counterparts, including a lighter color, a more fibrous and compact lobular pattern, and a frequent association with a sizable blood vessel near its medial aspect. The orbital fat can be considered an adynamic structure because its volume is not related to body habitus, and once removed it is not thought to regenerate.

\section{Innervation}

Sensory innervation to the lower lid derives mainly from the infraorbital nerve with minor contributions from the infratrochlear and zygomaticofacial nerve branches. The blood supply is obtained from the angular, infraorbital, and transverse facial arteries. Situated $2 \mathrm{~mm}$ below the ciliary margin, between the orbicularis oculi and the tarsus, is the marginal arcade, which should be avoided if a subciliary incision is used.

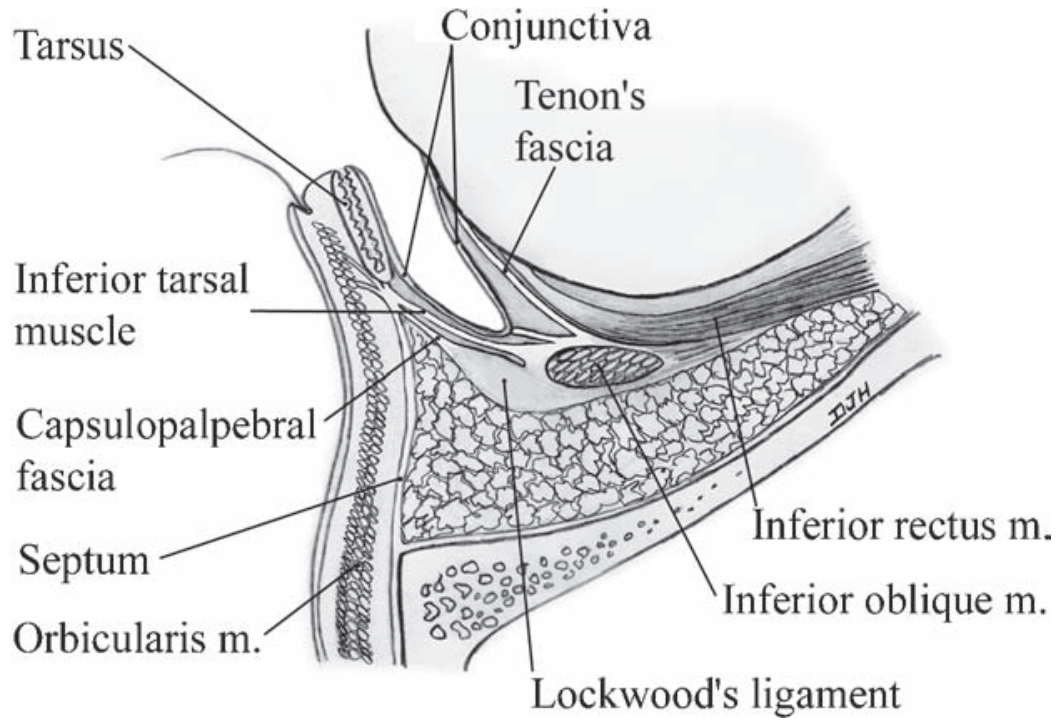

Fig. 23.3 Cross-sectional diagram of the lower eyelid demonstrating connective tissue expansion of inferior rectus into its terminal insertions. 


\section{Terminology}

Several descriptive terms are used pervasively in the literature of eyelid analysis and should be understood by those involved in surgical management of this area.

Blepharochalasis is a commonly misused term. It is an uncommon disorder of the upper eyelids, of unknown cause, which affects mainly young and middle-aged females. Blepharochalasis is characterized by recurrent attacks of painless unilateral or bilateral lid edema, causing a loss of skin elasticity and atrophic changes.

Dermatochalasis is an acquired condition of increased, abnormal laxity of the eyelid skin interrelated with genetic predisposition, natural aging phenomenon, and environmental influences. It is frequently associated with prolapsed orbital fat.

Steatoblepharon is a condition of true herniation or pseudoherniation of orbital fat behind a weakened orbital septum, causing areas of discrete or diffuse fullness in the eyelids. This condition and dermatochalasis are the two most common reasons why a patient presents to discuss eyelid surgery.

Festoons are single or multiple folds of orbicularis oculi in the lower lid that drape over onto themselves, creating an external, hammock-like bag. Depending on location, this bag may be preseptal, orbital, or jugal (cheek). It may contain fat.

Malar bags are areas of soft tissue fullness on the lateral edge of the infraorbital ridge and zygomatic prominence just superior to the palpebromalar sulcus. They are believed to be a result of recurrent dependent tissue edema and secondary fibrosis.

\section{Preoperative Evaluation}

A systematic and thorough preoperative assessment of blepharoplasty candidates is essential to minimize potential postoperative complications. Thus, patient analysis is directed to determining how much eyelid skin, orbicularis oculi, and orbital fat needs to be resected to optimize functional and aesthetic results, and whether the ocular and adnexal structures will tolerate such a surgical manipulation without undesirable sequelae.

\section{Risk Factors for Postoperative Dry Eye Syndrome}

Realizing that the protective physiological functions of blinking and eyelid closure are temporarily impaired following blepharoplasty, an appropriate ocular history should elicit information that might put the patient at greater risk for postoperative dry eye syndrome. Excessive tearing, a burning or gritty sensation, ocular discomfort, foreign bodies, mucus production, eyelid crusting, and a frequent need to blink are all symptoms suggestive of borderline or inadequate tear production. A possible atopic cause has to be ruled out.

Certain systemic diseases, particularly the collagen vascular diseases (i.e., systemic lupus erythematosus, scleroderma, periarteritis nodosa), Sjögren syndrome, Wegener granulomatosis, ocular pemphigoid, and StevensJohnson syndrome, can interfere with the glandular lubricating function and should be identified. ${ }^{6,7}$ The infiltrative ophthalmopathy of Graves' disease can result in vertical eyelid retraction and inadequate corneal protection after surgery and should be managed with medical treatment preoperatively and surgical conservatism during surgery. Thyroid hypofunction associated with a myxedematous state may mimic the baggy eyelids of dermatochalasis and should be ruled out. Incomplete recovery of a previous facial nerve insult may interfere with eyelid closure and predispose a patient to dry eye syndrome.

\section{Risk Factors for Postoperative Blindness}

Postoperative blindness, the most catastrophic complication of blepharoplasty, is associated with retrobulbar hemorrhage. ${ }^{8,9}$ Factors that influence bleeding tendencies should therefore be identified and controlled before surgery. ${ }^{10}$ Ingestion of aspirin, nonsteroidal antiinflammatory drugs (NSAIDS), antiarthritics, cortisone preparations, and vitamin $\mathrm{E}$ should be withheld for at least 14 days before surgery because of qualitative effects on platelet function. Over-the-counter medications should be discontinued as well because gingko biloba, for example, has been implicated in excessive bleeding. Similarly, St. John's wort is known to have hypertensive effects through a monoamine oxidase inhibitor mechanism. Warfarin compounds, if medically feasible, should be withheld to normalize the prothrombin time and may be reinstituted 48 to 72 hours after surgery.

Any history of abnormal or easy bruising, prolonged clotting time, or family members with bleeding dyscrasia should be noted with appropriate coagulation profiles. Hypertensive patients should be medically stabilized at least 2 weeks before surgery to ensure a nonfluctuating normotensive state. Women have a greater bleeding tendency during their menstrual period than at other cycle times, and this should be considered in planning surgery. Other important factors include drinking and smoking history, as the former (in large amounts) may influence platelet function, and the latter has been associated with delayed wound healing and diminished flap viability. Finally, any patient with a documented or suspected history of glaucoma must be evaluated and managed by an 
ophthalmologist to normalize intraocular pressures and guard against a potential acute closed-angle attack prior to any eyelid surgery. Some facial plastic surgeons recommend a routine ophthalmologic exam for all of their patients preoperatively.

\section{Ocular Assessment}

Examination of the eyes should begin with an overall inspection. The eyelid should be assessed for symmetry (by noting palpebral fissure height and length), position of the lower eyelid margin with respect to the inferior limbus, scleral show, and the presence of ectropion/entropion or exophthalmos/enophthalmos. Scars or skin lesions should be noted because it may be possible to include these in the resection. Areas of skin discoloration or abnormal pigmentation should also be noted.

Other baseline features of the periorbital area should be pointed out to the patient, particularly in lieu of the inability of blepharoplasty to correct them. ${ }^{11}$ The fine wrinkling and "crepe paper" appearance of the eyelid skin is not amenable to correction by blepharoplasty alone. Areas of abnormal pigmentation or discoloration (e.g., from venous stasis) will not be changed if outside the area of surgery and in fact may be more noticeable after surgery (because of light-refractile changes associated with conversion of a convex surface to a concave or flattened one). One of the greatest sources of dissatisfaction after lower blepharoplasty is the persistence of malar bags. The patient should realize that the upward tension necessary to attenuate such a soft tissue prominence would not be tolerated by the supporting structures of the lower lid and could lead to ectropion. Finally, lateral smile lines (crow's feet), despite the amount of lateral extension and undermining, are also not amenable to correction by standard blepharoplasty technique. All of these factors need to be discussed with the patient.

As a minimum, baseline ocular assessment should document visual acuity (i.e., best corrected vision if glasses or contact lenses are worn), extraocular movements, gross visual fields by confrontation, corneal reflexes, the presence of Bell's phenomenon and lagophthalmos. If there is any question of dry eye syndrome, the patient should be evaluated with Schirmer testing (to quantitate tear output) and tear film break-up times (to assess stability of precorneal tear film). ${ }^{12}$ Patients who demonstrate abnormalities in either or both of these tests or who have past or anatomical evidence that would predispose them to dry eye complications should be thoroughly evaluated by an ophthalmologist preoperatively. Also, a conservative skin-muscle excision (if not staged resection of upper and lower lids) would seem prudent.

\section{Assessment of Fat Pockets}

Evaluation of the adnexal structures should include assessment of the fat pockets. Palpation of the infraorbital rim is an essential component of this examination. A surgeon must recognize that a prominent rim limits the amount of orbital fat that can be removed without creating disparities in confluence between the lower eyelid and the anterior cheek. What appears to be an appropriate fat resection may contribute to a sunken-eye appearance if the patient has a very prominent rim. Assessment of the fat pockets may be facilitated by directing the patient's gaze in certain directions; a superior gaze accentuates the medial and central pockets, whereas looking upward and to the contralateral side accentuates the lateral pocket. Further confirmation of fatty prominence may be obtained by gentle retropulsion of the globe with the eyelid closed and observation of the outward movements of the respective fat pads.

\section{Assessment of Lid-Supporting Structures}

Because the most common cause of lower lid ectropion after blepharoplasty is failure to recognize a lax lower lid before surgery, it is essential to properly assess the lid-supporting structures. Two simple clinical tests aid in this evaluation. A lid distraction test (snap test) ${ }^{13}$ is performed by gently grasping the midportion of the lower eyelid between the thumb and index fingers and outwardly displacing the eyelid from the globe (Fig. 23.4). Movement of the lid margin greater than $10 \mathrm{~mm}$ indicates an abnormally lax supporting lid structure and suggests the need for a lid-shortening procedure. The lid retraction test $^{11}$ is used to assess lid tone as well as medial and lateral

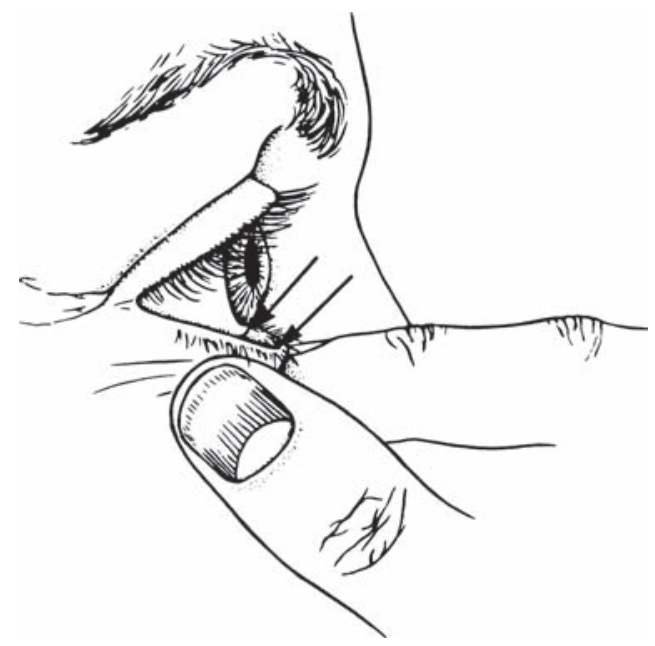

Fig. 23.4 Lid distraction test used to assess laxity of tarsoligamentous sling. (From Tenzel RR. Complications of blepharoplasty: orbital hematoma, ectropion, and scleral show. Clin Plast Surg 1981;8:799.) 

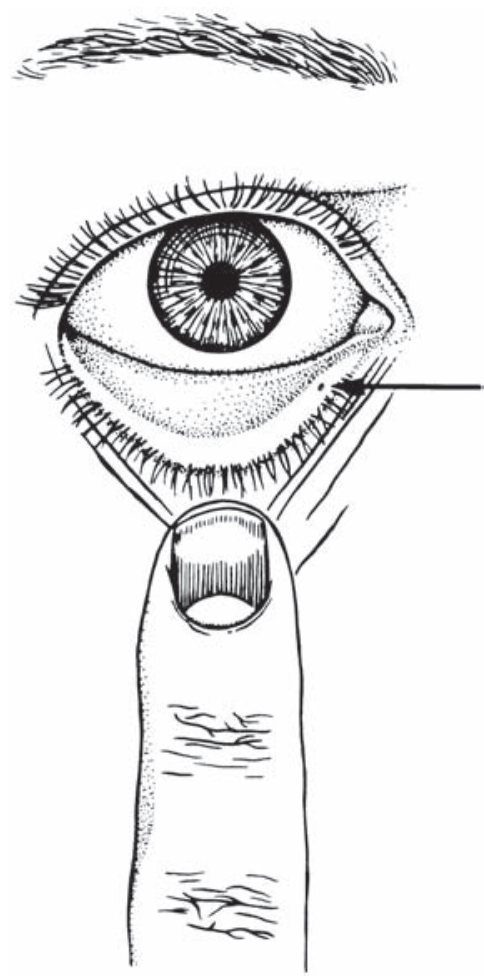

Fig. 23.5 Lid retraction test used to assess lower eyelid tone and stability of medial and lateral canthal tendon attachments. (From Tenzel RR. Complications of blepharoplasty: orbital hematoma, ectropion, and scleral show. Clin Plast Surg 1981;8:800.)

canthal tendon stability (Fig. 23.5). By using the index finger to inferiorly displace the lower lid toward the orbital rim, observations are made in terms of punctal or lateral canthal malposition (movement of puncta greater than $3 \mathrm{~mm}$ from the medial canthus indicates an abnormally lax canthal tendon and suggests the need for tendoplication). Releasing the eyelid, the pattern and rate of return of the lid to resting position should be observed. A slow return, or one that requires multiple blinks, indicates poor lid tone and eyelid support. Again, a conservative skinmuscle resection and lower lid-shortening procedure would be warranted.

\section{Surgical Technique}

Three basic surgical approaches have been described in lower lid blepharoplasty: (1) transconjunctival, (2) skinmuscle flap, and (3) skin flap.

\section{Transconjunctival Approach}

The transconjunctival approach to lower eyelid blepharoplasty was first described in 1924 by Bourquet. ${ }^{14}$ Although it is not a new procedure, over the past 10 years there has been a surge of interest and a growth of proponents for this approach. The transconjunctival lower lid blepharoplasty respects the integrity of the orbicularis oculi, an active support structure of the lower eyelid. This minimizes the incidence of ectropion. Also, an external scar can be avoided.

Proper patient selection for the transconjunctival approach is required. Ideal candidates include older patients with pseudoherniation of orbital fat and a limited amount of skin excess, young patients with familial hereditary pseudoherniation of orbital fat and no excess skin, all revision blepharoplasty patients, patients who do not want an external scar, patients with a history of keloids, and dark-skinned individuals who have a small possibility of hypopigmentation of the external scar. ${ }^{15,16}$ Because several authors have reported a significant reduction in short- and long-term complications with the transconjunctival approach to lower eyelid blepharoplasty compared with the skin-muscle method, the indications for the technique have been gradually expanding. ${ }^{15,17}$ The presence of excess lower lid skin does not preclude use of the transconjunctival approach. In the senior author's practice, the most commonly performed lower lid procedure consists of transconjunctival fat excision, pinch excision of skin, and 35\% trichloroacetic acid (TCA) peeling (described later). ${ }^{15,18-20}$ The skin excision is needed to recontour the lower eyelid once the fat has been removed. There frequently is less excess than one initially estimates before the fat excision is performed. ${ }^{17,21}$

\section{Preparation}

While sitting upright, the patient is asked to look upward. This helps to refresh the surgeon's memory as to which fat pads are the most prominent, and these are marked. The patient is then placed supine. Two drops of ophthalmic tetracaine hydrochloride $0.5 \%$ are then instilled into each inferior fornix. Prior to the local injections, our patients typically receive some intravenous sedation composed of midazolam (Versed) and meperidine hydrochloride (Demerol). Ten milligrams of intravenous dexamethasone (Decadron) is also given to help minimize postoperative edema. A local anesthetic mixture, consisting of equal parts of $0.25 \%$ bupivacaine (Marcaine) and 1\% lidocaine (Xylocaine) with 1:100,000 epinephrine to which is added a 1:10 dilution of sodium bicarbonate, is then injected into the lower lid conjunctiva using a 30 gauge needle. Experience has demonstrated that this mixture affords prolonged analgesic effect while minimizing the sting of initial infiltration through alkalinization of the local agent. The needle is advanced through the conjunctiva until the bony orbital rim is palpated. The local is slowly injected as the needle is withdrawn. This is performed medially, centrally, and laterally. Several surgeons also like to inject transcutaneously, although we have 


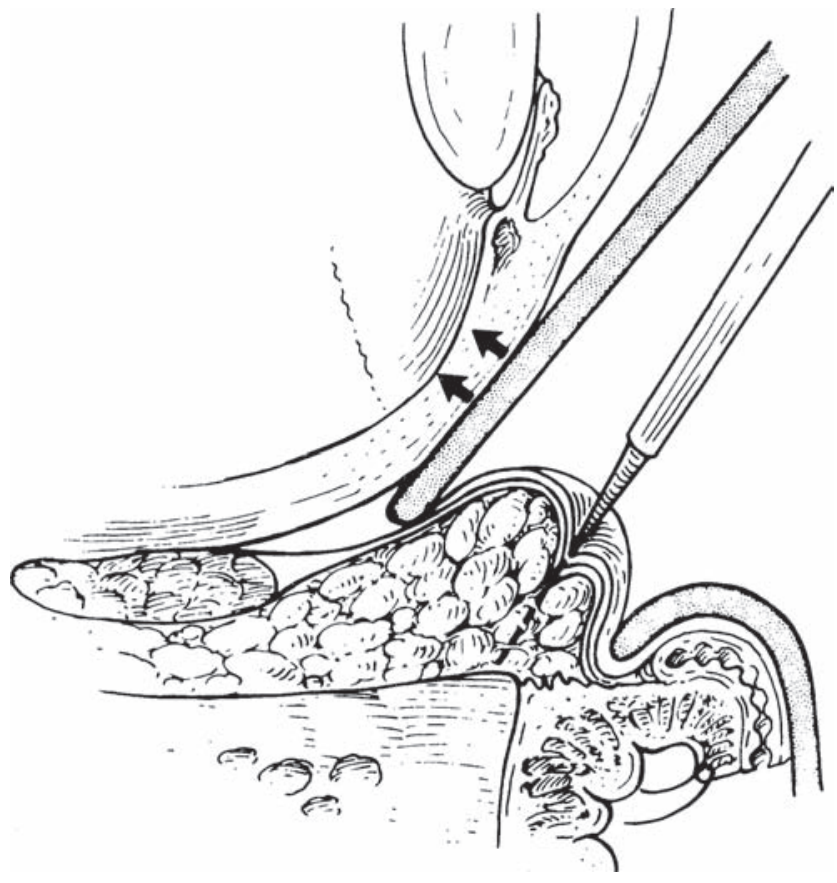

Fig. 23.6 Transconjunctival approach to lower eyelid fat pockets. Simultaneous eversion of the lower eyelid and ballottement of the globe produces a bulge of orbital fat that helps to guide the dissection. (From Baylis HI, Long JA, Groth MJ. Transconjunctival lower eyelid blepharoplasty: technique and complications. Ophthalmology 1989;96:1027.)

found that this is usually not necessary and may lead to unnecessary bruising.

\section{Incision}

After waiting a full 10 minutes for vasoconstriction to occur, the lower lid is gently retracted by an assistant using two small, double-pronged skin hooks (Figs. 23.6 and 23.7).

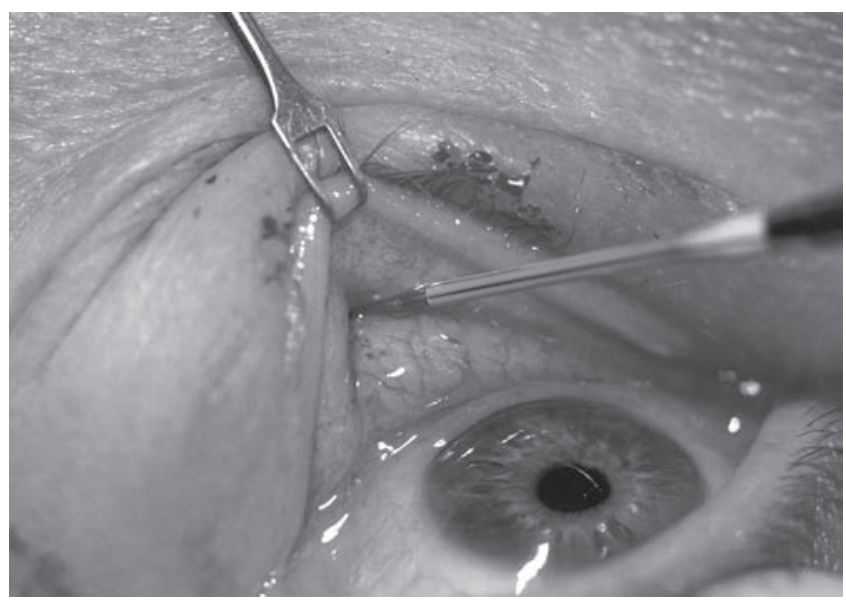

Fig. 23.7 The needle-tip bovie is poised to initiate the incision. (A corneal protector, or upper lid skin, is used for protection when the actual incision is made.)
The upper lid is placed over the globe to protect it. Either a guarded needle-tip bovie on a low setting or a no. 15 blade is used (others prefer a laser) to make the transconjunctival incision $2 \mathrm{~mm}$ below the inferior edge of the inferior tarsal plate. This inferior tarsal edge appears gray through the conjunctiva. The medial aspect of the incision is in line with the inferior punctum. The incision is carried just 4 to $5 \mathrm{~mm}$ shy of the lateral canthus.

Immediately after the transconjunctival incision is made, a single 5-0 nylon suture is placed in the conjunctiva closest to the fornix and used to retract the posterior lamella over the entire cornea (Fig. 23.8). Mosquitos snapped onto the patient's headwrap are used to hold the sutures under tension. The conjunctiva acts as a natural corneal protector and the superior retraction allows for easier plane dissection. The two skin hooks are then carefully removed and a Desmarres retractor is now used to evert the free edge of the lower lid (Fig. 23.9).

The distance of the transconjunctival incision from the inferior edge of the inferior tarsal plate determines whether one will approach the orbital fat preseptally or postseptally. ${ }^{16}$ We usually utilize the preseptal approach; therefore, our incisions are always $\sim 2 \mathrm{~mm}$ below the tarsus. The preseptal plane is an avascular plane between the orbicularis oculi and the orbital septum. ${ }^{22}$ Because the orbital septum is still intact while the preseptal plane is being developed, orbital fat does not bulge into one's view. The visualization obtained is closely similar to the orientation one is used to having when performing a skin-muscle

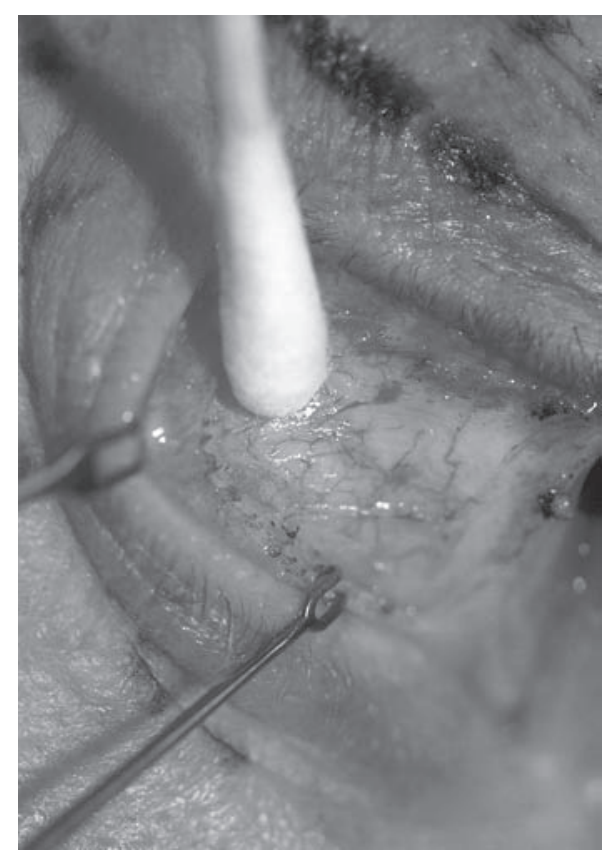

Fig. 23.8 Retraction suture in place; cotton-tipped applicator spreads incision site. 


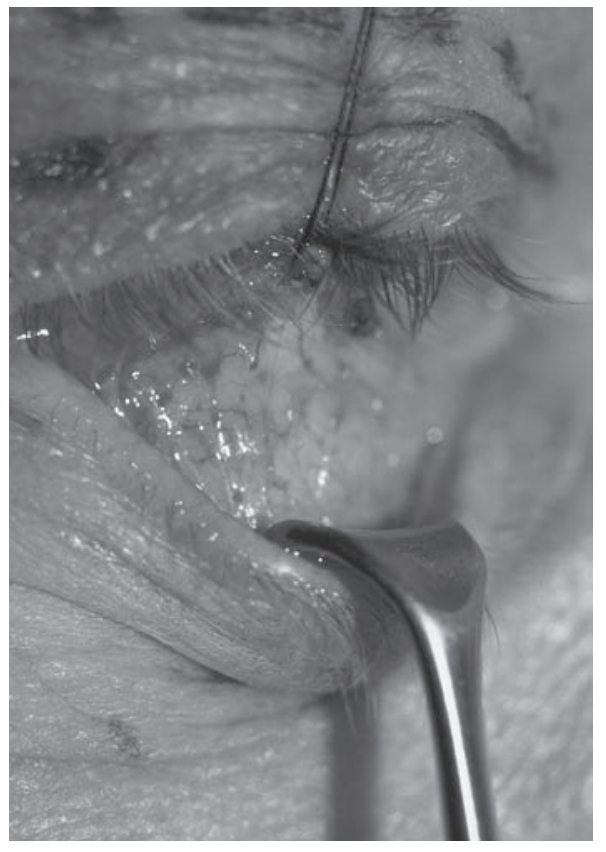

Fig. 23.9 Desmarres retractor exposes orbital septum.

flap blepharoplasty. The orbital septum will still have to be opened to access the orbital fat below (Fig. 23.10).

Others prefer the postseptal approach to the orbital fat. ${ }^{19}$ To directly access the fat pads the conjunctiva is incised $\sim 4 \mathrm{~mm}$ below the inferior border of the inferior tarsal plate and directly toward the anterior edge of the inferior orbital rim. The big advantage of this method is that the orbital septum is kept completely intact. Proponents for this technique state that the intact orbital septum adds to the support of the lower eyelid. One disadvantage is that the orbital fat immediately bulges into one's view. Care must be taken not to incise close to the conjunctival cul-de-sac to avoid the risk of synechiae. ${ }^{16}$ Also, the view

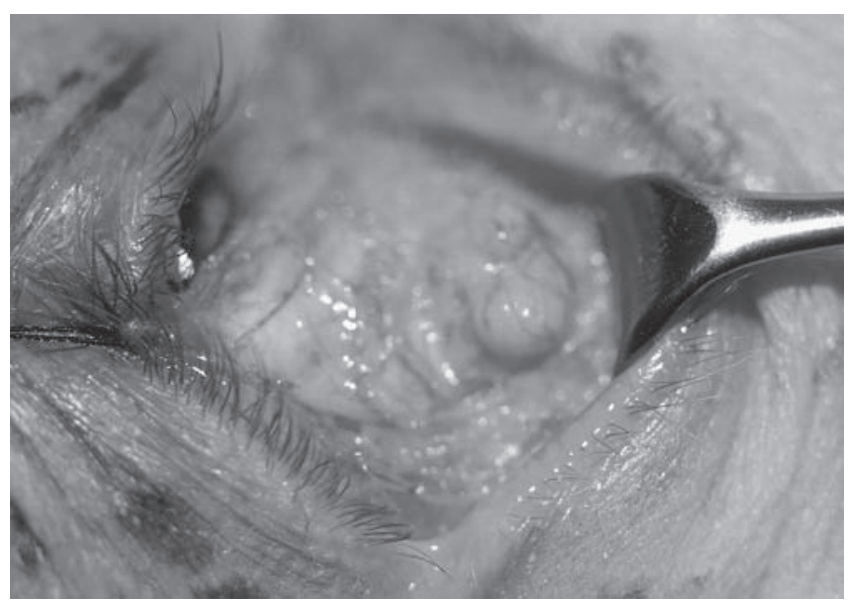

Fig. 23.10 Fat appearing through orbital septum. from the direct approach is one to which most facial plastic surgeons are less accustomed.

After the 5-0 suture retraction and Desmarres retractor are in place, the preseptal plane is developed with a combination of blunt dissection with a cotton swab and sharp dissection with scissors. It is mandatory to maintain a dry surgical field. Therefore, a bipolar cautery, "hot loop," or monopolar cautery is used to cauterize any bleeders.

The medial, central, and lateral fat pads are each individually identified through the septum with the help of some gentle digital pressure on the conjunctiva covering the globe. The orbital septum is then opened with scissors. Using forceps and a cotton-tipped applicator, the excess fat is carefully teased above the orbital rim and septum. Care must be taken to remove only the excessive and herniated fat because the eyes may take on a hollowed-out appearance following excessive fat excision. The ultimate goal is to achieve a lower eyelid contour that forms a smooth, gentle concave transition between it and cheek skin.

A 30 gauge needle is then used to inject a small amount of local anesthetic into the excess fat (Fig. 23.11). The bipolar cautery is used to cauterize across the fat stalk. When one is sure the entire stalk has been cauterized, scissors are used to cut across the cauterized area. Others, notably Cook, reduce fat volume with electrocautery, minimizing surgical excision. Many surgeons feel that the lateral fat pocket should be explored initially because its volume contribution becomes more difficult to assess after removal of its adjacent and interconnected central fat pad. ${ }^{11,23}$ After excess fat has been removed from each compartment, the field is examined to make sure there is no bleeding. Although $\mathrm{CO}_{2}$ laser fat excision has been advocated based on the advantages of hemostatic efficiency, precision, and reduced tissue trauma, the increased costs, requirements for highly trained personnel, and additional laser precautions have led us and others to abandon laser incisions for lower lid surgery. ${ }^{24,25}$

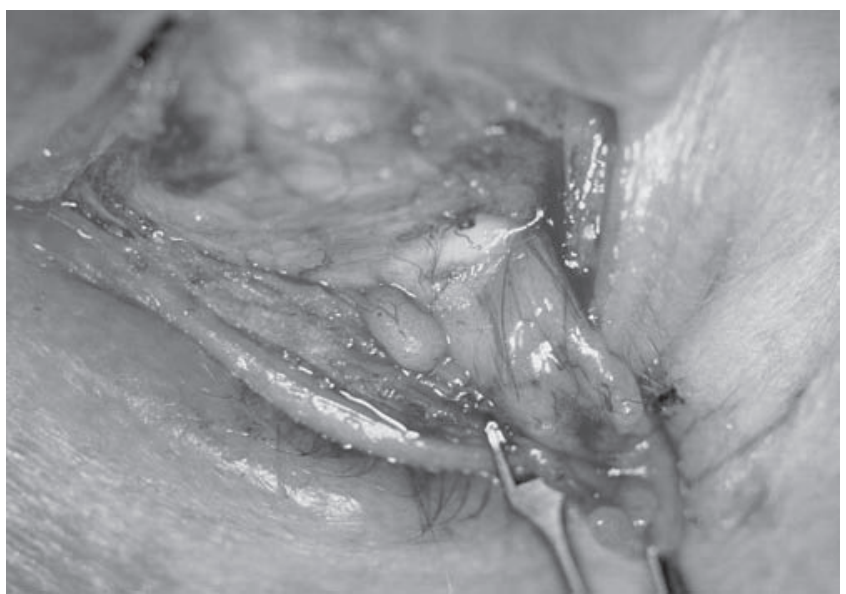

Fig. 23.11 Fat of medial compartment, partially excised. 
The Desmarres retractor should be removed periodically and the lower eyelid redraped over the fat that remains in place to facilitate examination of the contour of the eyelid. The fat that is removed is retained on a gauze on the surgical field in order from lateral to medial, allowing for comparison with the fat removed from the opposite side. For example, if preoperatively the surgeon felt that the right lateral fat pad was much larger than all others, then intraoperatively that compartment would have the most fat removed.

The medial and central fat compartments are separated by the inferior oblique muscle. This muscle must be clearly identified prior to the excision of excess fat from these compartments to prevent muscle injury. The medial fat pad is whiter than the central and lateral fat pads. This helps in its identification. The lateral compartment is usually isolated from the central one by a fascial band off the inferior oblique muscle. This fascial band can be cut safely.

After each successive fat compartment is treated, the entire field must again be examined for bleeding. After all of the bleeding has been cauterized with the bipolar, the Desmarres and the retraction sutures are removed. The lower lid is gently elevated upward and outward and then allowed to snap back into its proper position. This allows for proper realignment of the edges of the transconjunctival incision. No suture is required, although some surgeons feel more comfortable closing the incision with one, buried $6-0$ fast-absorbing gut stitch. Both eyes should then be irrigated with sodium chloride (ophthalmic Bbalanced salt solution).

In an older patient with skin excess, a lower lid skin pinch or chemical peel may now be performed. Using fixation forceps or Brown-Adson forceps, a 2 to $3 \mathrm{~mm}$ raised fold of redundant skin is raised just below the ciliary margin (Fig. 23.12). The skin fold is excised with sharp scissors,

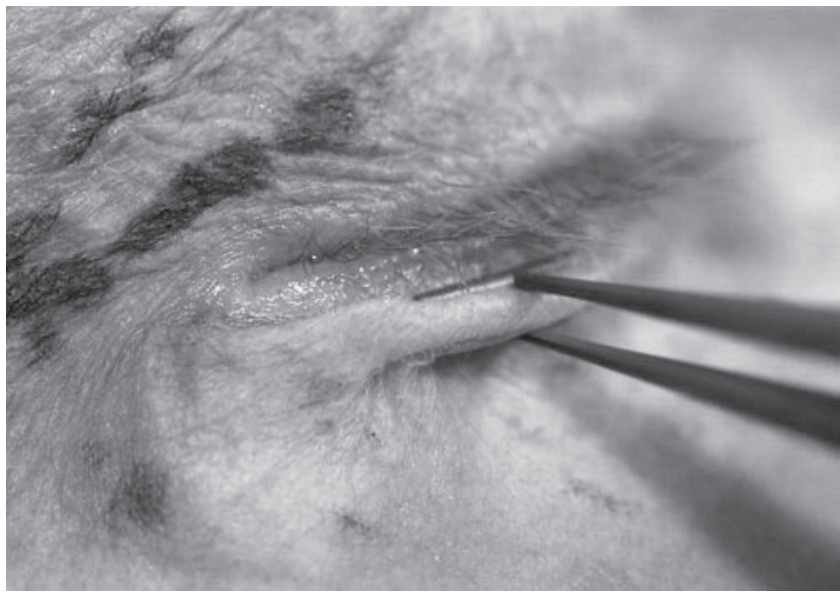

Fig. 23.12 Fixation forceps used to create ridge or mound for pinch excision.

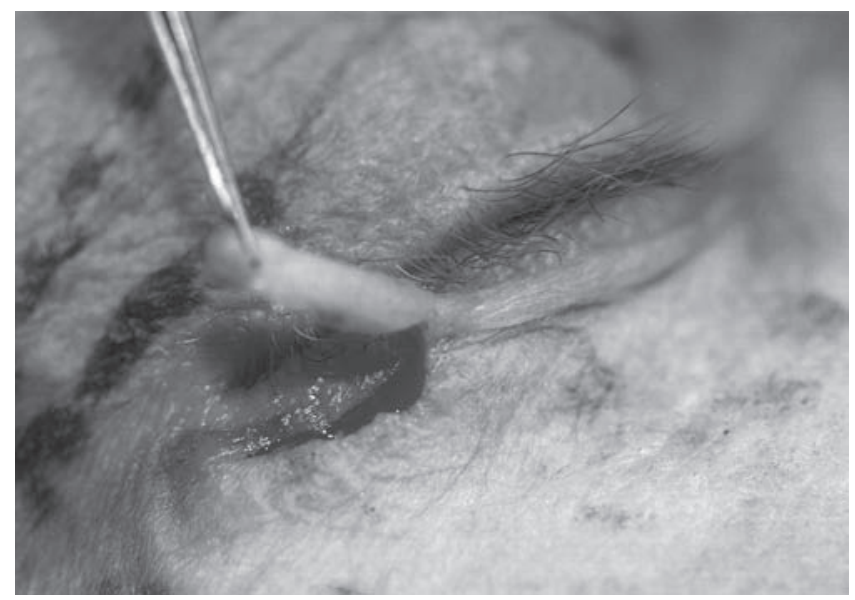

Fig. 23.13 Pinch excision progression.

with care taken not to cut the lower eyelashes (Fig. 23.13). The edges of the skin pinch are then brought together with interrupted $6-0$ fast-absorbing gut stitches. Several authors have closed this incision with cyanoacrylate (Histoacryl) or fibrin glue. ${ }^{15,26}$

Patients with crepey or fine lower eyelid rhytids are then treated with a 25 to 35\% TCA peel. The TCA is applied immediately below the skin pinch incision. A typical "frost" is generated (Fig. 23.14). Phenol is not used for lower lids in our hands because the erythema and inflammation phase is much longer than the TCA peel.

\section{Postoperative Care}

Immediately after surgery, the patient is kept quiet with head elevated at least 45 degrees. Cold compresses are placed on both eyes and changed every 20 minutes. The

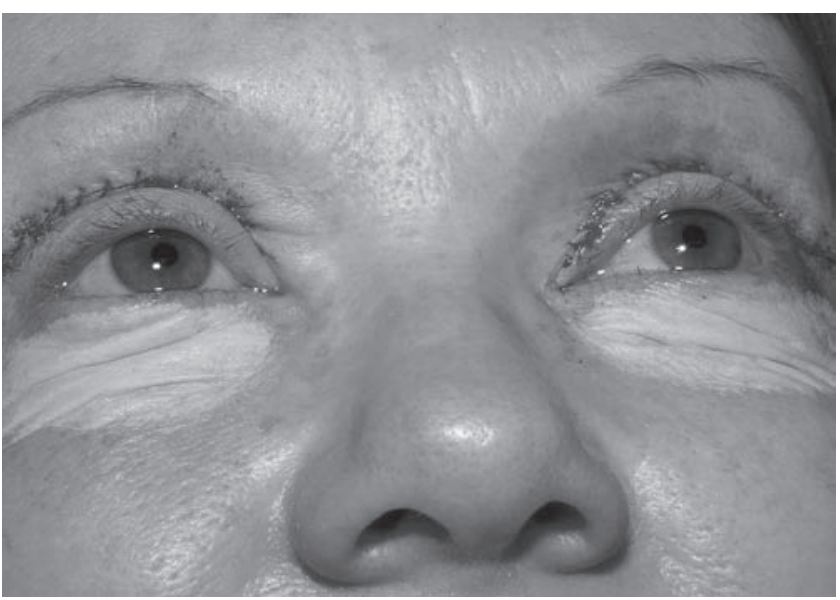

Fig. 23.14 Thirty percent trichloroacetic acid (TCA) lower lid peel frost. (Different patient in whom no pinch excision was done.) When the pinch technique is used, the TCA must not be applied any closer than $1 \mathrm{~mm}$ below the suture line. 
patient is observed closely for at least an hour for any signs of bleeding complications. The patient is given strict instructions to limit physical activity for the next week. The patient who is diligent about the cold compresses and head elevation during the first 48 hours will experience substantially less swelling. Some physicians place their patients on sulfacetamide ophthalmic drops during the first 5 postoperative days to help prevent an infection while the transconjunctival incision is healing.

\section{Skin-Muscle Flap Approach}

The skin-muscle flap approach was perhaps the most commonly used method in the 1970s and early 1980s. In patients with a large amount of excess skin and orbicularis oculi as well as fat pseudoherniation, this is an excellent procedure. The advantages of this approach are related to the safety and facility of dissecting in the relatively avascular submuscular plane and the ability to remove redundant lower eyelid skin. One must realize that even with the skin-muscle flap one is limited by how much skin can safely be removed without risking scleral show and even an ectropion. Persistent rhytids often remain despite attempts to safely resect redundant eyelid skin.

\section{Preparation}

Preparation for this method is similar to that for the transconjunctival approach, except that tetracaine drops are not necessary. A subciliary incision is planned 2 to $3 \mathrm{~mm}$ beneath the eyelid margin and is marked with a marking pen or methylene blue with the patient in the sitting position. Any prominent fat pads are also marked. The importance of marking the patient in the upright position before injection relates to the changes in soft tissue relationships that occur as a result of dependency and infiltration. The medial extent of the incision is marked $1 \mathrm{~mm}$ lateral to the inferior punctum to avoid potential damage to the inferior canaliculus, whereas the subciliary extension is carried to a point $\sim 8$ to $10 \mathrm{~mm}$ lateral to the lateral canthus (to minimize potential for rounding of the canthal angle and lateral scleral show). At this point, the lateral-most portion of the incision achieves a more horizontal orientation and is planned to lie within a crow's-foot crease line. Care should be exercised in planning the lateral extension of this incision to allow at least $5 \mathrm{~mm}$, and preferably $10 \mathrm{~mm}$, between it and the lateral extension of the upper blepharoplasty incision to obviate prolonged lymphedema.

Our patients typically receive intravenous sedation composed of midazolam and meperidine hydrochloride after the preoperative marking has been accomplished and intravenous dexamethasone is in. Before surgical prepping and sterile draping, the incision line (beginning laterally) and entire lower lid down to the infraorbital rim are infiltrated (superficial to orbital septum) with our anesthetic mixture previously described.

\section{Incision}

The incision, which is begun medially with a no. 15 scalpel blade, is only through skin to the level of the lateral canthus, but through skin and musculus orbicularis oculi lateral to this point. Using a blunt-tipped, straight-dissection scissors, the incision is undermined in a submuscular plane from lateral to medial and is then cut sharply by orientation of the blades in a caudal direction (optimizing the integrity of the pretarsal muscle sling). A Frost-type retention suture, using 5-0 nylon, is then placed through the tissue edge above the incision to aid in counterretraction. Using blunt dissection (with scissors and cotton-tipped applicators), a skinmuscle flap is developed down to, but not below, the infraorbital rim to avoid disruption of important lymphatic channels. ${ }^{27}$ Any bleeding points up to this point should be meticulously controlled with the handheld cautery or bipolar cautery, ${ }^{28}$ with conservatism exercised in the superior margin of the incision to avert potential thermal trauma to the eyelash follicles.

\section{Fat Removal}

If preoperative assessment suggests the need for fat-pad management, selective openings are made through the orbital septum over the areas of pseudoherniation and are guided by gentle digital pressure of the closed eyelid against the globe. Although alternatives aimed at electrocauterizing a weakened orbital septum exist ${ }^{29}$ that may obviate violation of this important barrier, we are comfortable with the long-term results and predictability of our technique of direct fat-pocket management.

After opening the septum (usually 5 to $6 \mathrm{~mm}$ above the orbital rim), the fat lobules are gently teased above the orbital rim and septum using forceps and a cotton-tipped applicator. The fat resection technique is very much as described in the transconjunctival technique and is not repeated.

Access to the medial compartment may be limited in part by the medial aspect of the subciliary incision. This incision should not be extended; instead, the fat should be gently teased into the incision, taking care to avoid the inferior oblique muscle. The medial fat pad is distinguished from the central pad by its lighter color.

\section{Closure}

In preparation for skin excision and closure the patient is asked to open the jaw widely and gaze in a superior 
direction. This maneuver creates a maximal voluntary separation of the wound edges and assists the surgeon in performing accurate resection of the skin-muscle flap. With the patient maintaining this position, the inferior flap is redraped over the subciliary incision in a superotemporal direction. At the level of the lateral canthus, the extent of skin muscle overlap is marked and incised vertically. A tacking stitch of 5-0 fast absorbing gut is then placed to maintain the position of the flap. Using straight scissors, the areas of overlap are conservatively resected (medial and lateral to the retention suture) so that edge-to-edge apposition can be maintained without the need for reinforcement. It is important to bevel the blades caudally to allow for a 1 to $2 \mathrm{~mm}$ strip resection of orbicularis oculi on the lower flap edge to avoid a prominent ridge at the time of closure. Some surgeons refrigerate the resected skin (viable for at least 48 hours) in sterile saline in case replacement tissue graft is needed after an overzealous resection eventuating in ectropion. It is far better to prevent such complications by performing a conservative resection.

After fat removal from the second eyelid, simple interrupted 6-0 fast-absorbing gut sutures are placed to close the incision on the initial eyelid. Attention can then be redirected back to redraping, trimming, and suturing the second eyelid. Finally, inch-long sterile strips are placed to aid in temporal support, and a light application of antibiotic ointment is applied to the sutured incision after irrigating the eyes with sodium chloride (balanced salt solution).

\section{Postoperative Care}

Postoperative care after the skin-muscle approach is essentially identical to the aftercare used in the transconjunctival approach. Bacitracin ophthalmic ointment is given to the patient for the subciliary incision. Iced saline compresses, head elevation, and limited activity are stressed to all patients.

\section{Skin Flap Approach}

The skin flap approach is perhaps the oldest and the least frequently used. This method allows independent resection and redraping of lower eyelid skin and underlying orbicularis oculi and is effective in repositioning and redraping excessively wrinkled, redundant, or deeply creased skin. ${ }^{30}$ In cases involving hypertrophy or festooning of the orbicularis oculi, direct access is provided for management, which allows for a greater and safer resection than would otherwise be tolerated if the flap were raised as a conjoined musculocutaneous unit. Disadvantages of this approach include a more tedious dissection that is associated with greater skin trauma (manifested by increased bleeding and eyelid induration), an increased risk of vertical eyelid retraction, and a higher demand placed on preoperative assessment of the fat pockets because of obscured subseptal observation by the overlying orbicularis oculi. ${ }^{31,32}$

The initial incision through skin is made only through the lateral extension of the subciliary marking to facilitate undermining. With an assistant maintaining downward traction on the lower eyelid skin (by placing a hand near the orbital rim), the lateral skin edge is grasped and pulled superiorly while sharp-scissors dissection carefully undermines the skin flap to a point just below the orbital rim. With the undermining accomplished, the subciliary incision is completed with the scissors. All bleeding points are precisely cauterized.

If the problem is skin redundancy or excessive wrinkling only, the skin flap is simply redraped in the manner described for skin-muscle flap. If access to the orbital fat compartments is required, these are approached by incising the orbicularis oculi $\sim 3$ to $4 \mathrm{~mm}$ inferior to the initial skin incision or via the transconjunctival approach. However, when orbicularis hypertrophy or festooning is present, optimal management is achieved by development of independent skin and muscle flaps. In this case, the muscle is incised (beveling caudally) across the extent of the incision, beginning $\sim 2 \mathrm{~mm}$ below the skin incision to preserve the pretarsal muscle sling. Undermining of the muscle flap is carried to just below the most dependent muscle roll (with festooning) or to a point that will allow effacement of a prominent muscle bulge (with hypertrophy) after muscle resection. After fat-pad management, the muscle flap is reinforced by suturing of its lateral end to orbital periosteum with 5-0 Vicryl (Ethicon, Inc., Somerville, NJ) and reapproximation of its pretarsal muscle edges with a few interrupted 5-0 chromic sutures. Again, skin closure follows the pattern previously described.

\section{Complications}

Complications after blepharoplasty are usually the result of overzealous skin or fat resection, lack of hemostasis, or an inadequate preoperative assessment. ${ }^{33,34}$ Less commonly, an individual's physiological response to wound repair may lead to undesirable sequelae despite execution of the proper technique. The goal in minimizing complications consequent to blepharoplasty must therefore focus on prevention by identifying and managing known risk factors.

\section{Ectropion}

One of the most common complications after lower lid blepharoplasty is eyelid malposition, which may range in presentation from a mild scleral show or rounding of 
the lateral canthal angle, to a frank ectropion with actual eyelid eversion. In most cases resulting in permanent ectropion, a failure to address excessive lower lid laxity is the etiologic culprit. Other causes include excessive skin or skin-muscle excisions, inferior contracture along the plane of the lower lid retractors and orbital septum (greater in skin flap technique), inflammation of the fat pockets, and, rarely, destabilization of the lower lid retractors (a potential yet uncommon complication of the transconjunctival approach). Temporary ectropion has been associated with lid loading from reactionary edema or hematoma and muscle hypotonicity.

A conservative approach to management may include the following: (1) a short course of perioperative steroids with cold compresses and head elevation to manage edema; (2) warm and cool compresses alternated to hasten resolution of minor established hematomas and improve circulatory status; (3) repeated squinting exercises to improve muscle tonus; (4) gentle massage in an upward direction; and (5) supportive taping of the lower lid (upward and outward) to assist in corneal protection and tear collection.

When skin excisions are recognized to be excessive within the first 48 hours, the banked eyelid skin should be used as a replacement graft. If recognition is delayed, conservative measures to protect the eye should be used to allow the scar to mature and a full-thickness graft (preferably upper eyelid skin or, alternatively, postauricular skin, or foreskin in males) used to replace the deficit. In many cases, a lid-shortening procedure is combined with the tissue grafting and is the mainstay of treatment when an atonic lid is present. Management of persistent indurations, resulting from hematoma formation or inflammatory responses of the fat pockets, generally involves direct depot injections of corticosteroid.

\section{Hematomas}

Collections of blood beneath the skin surface can usually be minimized before surgery by optimizing coagulation profiles and normotensive status during surgery through delicate tissue handling and meticulous hemostasis and after surgery through head elevation, cold compressing, a controlled level of activity, and appropriate analgesic support. Should a hematoma develop, its extent and time of presentation will guide management.

Small, superficial hematomas are relatively common and are typically self-limiting. If organization occurs with the development of an indurated mass and resolution is slow or nonprogressive, conservative steroid injections may be used to hasten the healing process. Moderate or large hematomas recognized after several days are best managed by allowing the clot to liquify ( 7 to 10 days) and then evacuating the hematoma through large-bore needle aspiration or by creating a small stab wound over it with a no. 11 blade. Hematomas that are large and present early, that are expanding, or that represent symptomatic retrobulbar extension (decrease in visual acuity, proptosis, ocular pain, ophthalmoplegia, progressive chemosis) demand immediate exploration and hemostatic control. In the case of the latter, urgent ophthalmologic consultation and orbital decompression are the mainstays of treatment.

\section{Blindness}

Blindness, though rare, is the most feared potential complication of blepharoplasty. It occurs with an incidence of $\sim 0.04 \%,{ }^{35}$ typically presents itself within the first 24 hours after surgery, and is associated with orbital fat removal and the development of a retrobulbar hematoma (medial fat pocket most commonly involved). Commonly implicated causes of retrobulbar hemorrhage include the following: (1) excessive traction on orbital fat resulting in disruption of small arterioles or venules in the posterior orbit; (2) retraction of an open vessel beneath the septum after fat release; (3) failure to recognize an open vessel because of vasospasm or epinephrine effect; (4) direct vessel trauma resulting from injections done blindly beneath the orbital septum; and (5) rebleeding after closure resulting from any maneuver or event that leads to an increased ophthalmic arteriovenous pressure head.

Early recognition of a developing orbital hematoma can be facilitated by delaying intraoperative closure (first side), avoiding occlusive-pressure eye dressings, and extending the postoperative observational period. Although many methods of management have been described to manage threatened vision resulting from elevated intraocular pressures (reopening the wound, lateral canthotomy, steroids, diuretics, anterior chamber paracentesis), the most effective definitive treatment is immediate orbital decompression, which is usually accomplished through medial wall and orbital floor resections. ${ }^{8,36}$ Certainly, ophthalmologic consultation is advisable.

\section{Epiphora}

Assuming dry eye syndrome was ruled out before surgery or managed appropriately intraoperatively (conservative and staged resections), a dysfunctional lacrimal collecting system rather than a high glandular output state is typically responsible for postoperative epiphora (although reflex hypersection may be a contributing factor because of coexistent lagophthalmos or vertical retraction of the lower lid). This response is common in the early postoperative period and is usually self-limited. Causes include the following: (1) punctal eversion and canalicular distortion secondary to wound retraction and edema; (2) impairment 
of the lacrimal pump resulting from atony, edema, hematoma, or partial resection of the orbicularis oculi sling; and (3) a temporary ectropion resulting from lid loading. Outflow obstructions, secondary to a lacerated inferior canaliculus, are preventable by keeping the lower lid incision lateral to the punctum. Should laceration injury occur, primary repair over a Silastic stent (Crawford tube; Dow Corning, Midland, MI) is recommended. Persistent punctal eversion can be managed by cauterization or diamond excision of the conjunctival surface below the canaliculus.

\section{Suture Line Complications}

Milia or inclusion cysts are common lesions seen along the incisional line resulting from trapped epithelial debris beneath a healed skin surface or possibly from the occlusion of a glandular duct. They are typically associated with simple or running cuticular stitches. Their formation is minimized by subcuticular closure. If they develop, definitive therapy is aimed at uncapping the cyst (no. 11 blade or epilation needle) and teasing out the sac. Granulomas may develop as nodular thickenings within or beneath the suture line and are typically treated by steroid injections if small and by direct excision if large. Suture tunnels develop as a result of prolonged suture retention and epithelial surface migration along the suture tract. Preventive treatment includes early suture removal (3 to 5 days), and definitive treatment involves unroofing the tunnel. Suture marks are also related to prolonged suture retention and their formation can usually be avoided by using a rapidly absorbing suture (fast-absorbing gut or mild chromic), by removing a monofilament suture early, or by employing a subcuticular closure.

\section{Wound Healing Complications}

Although rare, hypertrophic or prominent lower eyelid scars may develop because of improper placement of the lower lid incision. If extended too far medially in the epicanthal region, bow-string or web formation may occur (conditions usually amenable to correction by Z-plasty technique). A lateral canthal extension (which normally overlies a bony prominence) that is oriented too obliquely downward or is closed under excessive tension predisposes an incision to hypertrophic scarring, and during healing the vertical contraction vectors act on the lateral lid to favor scleral show or eversion. If the lower lid incision is oriented too far superiorly or too close to the lateral aspect of the upper lid incision, the forces of contraction (now favoring a downward pull) provide conditions that predispose the patient to lateral canthal hooding. Again, proper treatment should be aimed at reorienting the direction of contracting vectors.
Wound dehiscence may develop as a result of closure under excessive tension, early removal of sutures, extension of an infectious process (unusual), or hematoma (more commonly). Skin separation is seen most often in the lateral aspect of the incision with the skin-muscle and skin techniques, and treatment is directed to supportive taping or resuturing. If tension is too great for conservative management, then a lid suspension technique and lateral grafting should be considered. Skin slough may develop as a result of devascularization of the skin segment. It is almost exclusively seen in the skin-only technique and typically occurs in the lateral portion of the lower eyelid after wide undermining and subsequent hematoma formation. Treatment consists of local wound care, evacuation of any hematomas, establishment of a line of demarcation, and early skin replacement to obviate scar contracture of the lower lid.

\section{Skin Discoloration}

Areas of skin undermining are frequently evident as hyperpigmentation in the early recovery period secondary to bleeding beneath the skin surface with subsequent hemosiderin formation. This process is usually self-limiting and often takes longer to resolve in darkly pigmented individuals. It is imperative during the healing process, and particularly in this patient population, to avoid direct sunlight because this may lead to permanent pigment changes. Refractory cases (after 6 to 8 weeks) may be considered for camouflage, periorbital peeling, or depigmentation therapy (e.g., hydroxyquinone, kojic acid). Telangiectasias may develop after skin undermining, particularly in areas beneath or near the incision, and most commonly occur in patients with preexisting telangiectasias. Treatment options may include chemical peeling or dye laser ablation.

\section{Ocular Injury}

Corneal abrasions or ulcerations may result from inadvertent rubbing of the corneal surface with a gauze sponge or cotton applicator, instrument or suture mishandling, or desiccation developing as a result of lagophthalmos, ectropion, or preexistent dry eye syndrome. Symptoms suggestive of corneal injury, which include pain, eye irritation, and blurred vision, should be confirmed by fluorescein staining and slitlamp examination by an ophthalmologist. Therapy for mechanical injury typically involves use of an antibiotic ophthalmic drop with lid closure until epithelialization is complete (usually 24 to 48 hours). Treatment for dry eye syndrome includes the addition of ocular lubricants, such as Liquitears and Lacri-lube.

Extraocular muscle imbalance, manifested by gaze diplopia, may be seen and is often transitory, presumably 
reflecting resolution of an edematous process. However, permanent muscle injury may result from blind clamping, deep penetration of the fat pockets during sectioning of the pedicle, thermal injury resulting from electrocauterization, suture incorporation during closure, or ischemic contracture of the Volkman type. Patients with evidence of refractory and incomplete recovery of muscle function should be referred to an ophthalmologist for evaluation and definitive treatment.

\section{Contour Irregularities}

Contour irregularities are generally caused by technical omissions. Overzealous fat resection, particularly in a patient with a prominent infraorbital rim, results in a lower lid concavity and contributes to a sunken-eye appearance. Failure to remove enough fat (common in lateral pocket) leads to surface irregularities and persistent bulges. A ridge that persists beneath the incision line is usually the result of inadequate resection of a strip of orbicularis oculi before redraping. Areas of induration or lumpiness below the suture line usually can be attributed to unresolved or organized hematoma, tissue reaction or fibrosis secondary to electrocauterization or thermal injury, or soft tissue response to fat necrosis. Treatment in each case is directed at the specific cause. Persistent fat bulges are managed by resection, whereas areas of lid depression can be managed by sliding fatpad grafts, free-fat or dermal fat grafts, ${ }^{37}$ or orbicularis oculi flap repositioning. Some patients with such bulges or prominences respond to direct injections of triamcinoline $\left(40 \mathrm{mg} / \mathrm{cm}^{3}\right)$. In selected cases, infraorbital rim reductions distract noticeability from a hollow-eye appearance and may be used as an adjunctive technique. Unresolved hematomas and areas of heightened inflammatory response may be managed with conservative injections of steroids.

\section{References}

1. Zide BM. Anatomy of the eyelids. Clin Plast Surg 1981;8:623

2. Aguilar GL, Nelson C. Eyelid and anterior orbital anatomy. In: Hornblass A, ed. Oculoplastic, Orbital and Reconstructive Surgery. Vol. 1: Eyelids. Baltimore: Williams \& Wilkins; 1988

3. Jones LT. New concepts of orbital anatomy. In: Tessier P, Callahan A, Mustarde JC, et al, eds. Symposium on Plastic Surgery in the Orbital Region. St Louis: CV Mosby; 1976

4. Doxanas MT. Blepharoplasty: key anatomical concepts. Facial Plast Surg 1984; $1: 259$

5. Nesi F, Lisman R, Levine M. Smith's Ophthalmic Plastic and Reconstructive Surgery. 2nd ed. St. Louis: CV Mosby; 1998:1-78

6. Rees TD, Jelks GW. Blepharoplasty and the dry eye syndrome: guidelines for surgery? Plast Reconstr Surg 1981;68:249

7. Jelks GW, McCord CD. Dry eye syndrome and other tear film abnormalities. Clin Plast Surg 1981;8:803
8. Sacks SH, Lawson W, Edelstein D, et al. Surgical treatment of blindness secondary to intraorbital hemorrhage. Arch Otolaryngol Head Neck Surg 1988;114:801

9. Mahaffey PJ, Wallace AF. Blindness following cosmetic blepharoplasty: a review. Br J Plast Surg 1986;39:213

10. Callahan MA. Prevention of blindness after blepharoplasty. Ophthalmology 1983;90:1047-1051

11. Beekhuis GJ. Blepharoplasty. Otolaryngol Clin North Am 1982;15: 179

12. McKinney P, Zukowski ML. The value of tear film breakup and schirmer's tests in preoperative blepharoplasty evaluation. Plast Reconstr Surg 1989;84:572

13. Holt JE, Holt GR. Blepharoplasty: indications and preoperative assessment. Arch Otolaryngol 1985;111:394

14. Bourquet J. Les hernies graisseuses de l'orbite: notre traitment chirurgical. Bull Acad Natl Med 1924;92:1270-1272

15. Perkins SW, Dyer WD II, Simo F. Transconjunctival approach to lower eyelid blepharoplasty. Arch Otolaryngol Head Neck Surg 1994;120: 172-177

16. Mahe E. Lower lid blepharoplasty: the transconjunctival approach: extended indications. Aesthetic Plast Surg 1998;22:1-8

17. Zarem HA, Resnick JI. Minimizing deformity in lower blepharopasty: the transconjuctival approach. Plast Reconstr Surg 1991;88:215

18. McKinney P, Zukowshi ML, Mossie R. The 4th option: a novel approach to lower lid blepharoplasty. Aesthetic Plast Surg 1991;15: 293-296

19. Baylis HI, Long JA, Groth MJ. Transconjunctival lower eyelid blepharoplasty. Ophthalmology 1989;96:1027

20. Cheney ML. Facial Surgery: Plastic and Reconstructive. Baltimore: Williams \& Wilkins; 1987:895-904

21. Netscher DT, Patrinely JR, Peltier M, et al. Transconjunctival versus transcutaneous lower eyelid blepharoplasty: a prospective study. Plast Reconstr Surg 1995;96:1053-1059

22. Tessier P. The conjunctival approach to the orbital floor and maxilla in congenital malformation and trauma. J Maxillofac Surg 1973;1:3-8

23. Spira M. Blepharoplasty. Clin Plast Surg 1978;5:121

24. David LM. The laser approach to blepharoplasty. J Dermatol Surg Oncol 1988; $14: 741$

25. Mele JA III, Kulick MI, Lee D. Laser blepharoplasty: is it safe? Aesthetic Plast Surg 1998;22:9-11

26. Mommaerts MY, Beirne JC, Jacobs WI, Abeloos JSV. Use of fibrin glue in lower blepharoplasties. J Craniomaxillofac Surg 1996;24:78-82

27. Holt JE, Holt GR, Cortez EA. Blepharoplasty. Ear Nose Throat J $1981 ; 60: 42$

28. Robinson L, Crumley RL. Electrocoagulation in blepharoplasty: experi- Q2 mental data in the rabbit, 1990; unpublished.

29. Cook TA, Dereberry J, Harrah ER. Reconsideration of fat pad management in lower lid blepharoplasty surgery. Arch Otolaryngol 1984;110:521

30. Klatsky SA, Manson PN. Separate skin and muscle flaps in lower lid blepharoplasty. Plast Reconstr Surg 1981;67:151

31. Wolfey DE. Blepharoplasty: the ophthalmologist's view. Otolaryngo Clin North Am 1980;13:237

32. McCollough EG, English JL. Blepharoplasty: avoiding plastic eyelids. Arch Otolaryngol Head Neck Surg 1988;114:645

33. Adams BJS, Feurstein SS. Complications of blepharoplasty. Ear Nose Throat J 1986;65(1):11-28

34. Castanares S. Complications in blepharoplasty. Clin Plast Surg 1978;5:149

35. Moser MH, DiPirro E, MaCoy FJ. Sudden blindness following blepharoplasty: report of seven cases. Plast Reconstr Surg 1973;51:363

36. Anderson RL, Edwards JJ. Bilateral visual loss after blepharoplasty. Ann Plast Surg 1980;5:288

37. Loeb R. Fat pad sliding and fat grafting for leveling lid depressions. Clin Plast Surg 1981;8:757 


\section{Author Query}

Q1: AU: Mosquito clips? unclear.

Q2: AU: Ref 28: unpublished material can not be listed in the references. It must be cited in text in parens, such as: (Robinson L, Crumley RL, unpublished data, 1990). Please move citation to text. Can you provide a substitute reference for Ref 28? If no, delete ref number from text and list and re-number going forward in text and in ref list. 